$\begin{array}{llll}\text { Submission: 29/01/2019; } & 1^{\text {st }} \text { round notif.: 23/03/2019; } & \text { New version: 16/04/2019; } & 2^{\text {nd }} \text { round notif.: 15/08/2019 } \\ \text { Camera ready: 22/09/2019; } & \text { Edition review: 22/12/2019; } & \text { Available online: 16/02/2020; } & \text { Published: 16/02/2020 }\end{array}$

\title{
Uma abordagem de design para MOOC: um mapeamento sistemático da articulação entre design instrucional e de interação
}

\author{
Title: A design approach for MOOC: a systematic mapping of the articulation between \\ instructional and interaction design
}

\author{
Filipe Adeodato Garrido \\ Instituto de Matemática e \\ Estatística (IME) - \\ Departamento de Ciência \\ da Computação (DCC) - \\ Universidade Federal da \\ Bahia (UFBA) \\ filipe.garrido@ufba.br
}

\author{
Beatriz Brito do Rêgo \\ Instituto de Matemática e \\ Estatística (IME) - \\ Departamento de Ciência \\ da Computação (DCC) - \\ Universidade Federal da \\ Bahia (UFBA) \\ beatrizbr@ufba.br
}

\author{
Rita Suzana P. Maciel \\ Instituto de Matemática e \\ Estatística (IME) - \\ Departamento de Ciência \\ da Computação (DCC) - \\ Universidade Federal da \\ Bahia (UFBA) \\ rita.suzana@ufba.br
}

\author{
Ecivaldo de Souza Matos \\ Instituto de Matemática e \\ Estatística (IME) - \\ Departamento de Ciência \\ da Computação (DCC) - \\ Universidade Federal da \\ Bahia (UFBA) \\ ecivaldo@ufba.br
}

\section{Resumo}

MOOC é um acrônimo para Massive Open Online Course, uma modalidade de educação online que oferta cursos gratuitos a diversos públicos, com diferentes idades, formações e nacionalidades. Acredita-se que abordagens de design que integrem design de interação e o design instrucional podem favorecer a comunicabilidade em MOOC, refletindo positivamente na aprendizagem do estudante. Nesse sentido, este artigo possui como objetivo apresentar os resultados de um Mapeamento Sistemático de Literatura (MSL). Em sua metodologia esse mapeamento e buscou identificar estudos e pesquisas primárias referentes a processos de design de interação e de design instrucional aplicados a MOOC. Os resultados encontrados apontam para a existência de desafios de pesquisa de articulação teórica e metodológica entre o design de interação e design instrucional em MOOC. Os resultados apresentados neste artigo apontam a inexistência de uma articulação conjunta entre as áreas e sustentam a relevância de uma abordagem híbrida de design (design de interação articulado ao design instrucional) focalizada em MOOC.

Palavras-Chave: MOOC; design de interação; design instrucional;

\begin{abstract}
MOOC is an acronym for Massive Open Online Course, an online education modality that offers free courses to diverse audiences with different ages, backgrounds and nationalities. It is believed that design approaches that integrate interaction design and instructional design may favor communicability in MOOC, positively reflecting student learning. In this sense, this article aims to present the results of a Systematic Literature Mapping (SLM). In methodology in this mapping sought to identify studies and primary research related to interaction design and instructional design processes applied to the MOOC. The results found point to the existence of research challenges of theoretical and methodological articulation between interaction design and instructional design in MOOC. The results presented in this article point to the lack of a joint articulation between the areas and support the relevance of a hybrid design approach (articulated interaction design to instructional design) focused on MOOC.
\end{abstract}

Keywords: MOOC; interaction design; instructional design.

Cite as: Garrido, F. A., Do Rêgo, B. B., Maciel, R. S. P., \& Matos, E. (2020). A design approach for MOOC: a systematic mapping of the articulation between instructional and interaction design. (Uma abordagem de design para MOOC: um mapeamento sistemático da articulação entre design instrucional e de interação). Brazilian Journal of Computers in Education (Revista Brasileira de Informática na Educação - RBIE), 28, 115-132. DOI: 10.5753/RBIE.2020.28.0.115 


\section{Introdução}

O termo MOOC surgiu em 2008 por intermédio dos professores Dave Cormier da University of Prince Edward Island, e Bryan Alexander do National Institute for Technology in Liberal Education, sendo um acrônimo para Massive Open Online Course (Curso Online Massivo Aberto) [Grainger 2013]. Segundo Mattar (2013), MOOC é um tipo de curso online ${ }^{1}$, aberto (gratuito e sem pré-requisitos para participação) e massivo (disponível a um grande número de alunos e com vasto material); podendo ser instanciado em diferentes tipos de plataformas web, para além dos tradicionais ambientes virtuais de aprendizagem, como o Moodle.

Os MOOC têm sido considerados uma das tendências mais proeminentes para o ensino superior dos últimos anos [Baturay 2015]. Esse fato se deve ao grande número de instituições de ensino superior que possuem plataformas próprias ou parceria com outras instituições para lançar cursos para o público externo ao campus [Matta e Figueiredo 2013, Boall et al. 2015, Dal Forno e Knoll 2013, Rodrigues et al. 2016b].

A pluralidade de perfis, necessidades e interesses de pessoas que procuram cursos em MOOC, aliada à quantidade massiva de acessos às plataformas de MOOC, representa um desafio de pesquisa para a comunidade de pesquisa em Informática na Educação, especialmente na concepção de propostas de design de MOOC [Fassbinder et al. 2014], seja no aspecto de interação, sob a perspectiva do Design de Interação; seja no aspecto da abordagem pedagógica/andragógica, sob a perspectiva do Design Instrucional/Educacional.

Nesse sentido, este artigo apresenta resultados de um Mapeamento Sistemático de Literatura (MSL), cujo objetivo foi identificar estudos e pesquisas primárias referentes a processos de design de interação e de design instrucional aplicados a MOOC. Esses resultados já explicitam alguns problemas e algumas soluções, por meio de técnicas, métodos ou abordagens de design de interação ou design instrucional focalizados em MOOC.

O texto a seguir está estruturado em quatro seções, a citar: fundamentação teórica, a metodologia aplicada na condução do MSL, os resultados e, por fim, as considerações e trabalhos futuros originados deste MSL.

\section{Fundamentação teórica}

O projeto de design de sistemas computacionais interativos e o planejamento das interações dos usuários sobre estes sistemas é tema de estudo do Design de Interação (DI) [Rogers et al. 2013]. Todavia, para Löwgren, o DI não é necessariamente uma área, mas o processo de modelagem das artefatos digitais para o uso humano [Löwgren 2013]. Neste artigo, consideramos a definição de Löwgren por favorecer a relação conceitual entre o DI e o design instrucional.

Segundo Filatro, o Design Instrucional (DInst) é um processo de concepção curricular ${ }^{2}$ em qualquer nível ou modalidade educacional, com o objetivo de produzir resultados de aprendizagem específicos; baseados não apenas na pesquisa pedagógica, mas também em práticas educacionais contemporâneas [Filatro e Piconez 2004].

\footnotetext{
${ }^{1}$ Ressaltamos que neste estudo os MOOC são tratados como abordagem pedagógica; portanto, não o referenciamos como plataformas ou sistemas computacionais.

2 Inventário de atividades implementadas com vistas a conceber, organizar e planejar uma ação educacional ou de formação, incluindo a definição de objetivos, conteúdos, métodos (incluindo avaliação) e materiais de aprendizagem, bem como disposições para a formação de professores e formadores (CEDEFOP - European Centre for the Development of Vocational Training, 2011).
} 
Acredita-se que unificar práticas dessas duas áreas, DI e DInst, na concepção de MOOC pode favorecer a redução de custos de manutenção de cursos, a integração dos discurso de metacomunicação da interface, reduzir ruídos de comunicação entre os membros da equipe técnico-pedagógica na concepção dos cursos, além de agilizar processos ora realizados separadamente.

Segundo Baloi (2009), os MOOC têm por objetivo o compartilhamento de diferentes experiências individuais, em que o estudante, com aquilo que aprendeu em casa, ao se encontrar com os outros, se enriquece com a sua experiência, ocorrendo assim relações de inter-ajudas recíprocas em que cada um aprende com o outro, entendendo que se deve respeitar a experiência individual de cada sujeito.

Espera-se que articulação entre design de interação e design instrucional, focalizada na comunicação usuário-sistema e usuário-sistema-usuário, possa mitigar eventuais rupturas de comunicabilidade e favorecer a construção colaborativa de conhecimento [Garrido 2018].

\subsection{Trabalhos correlatos}

Foram encontrados quatro trabalhos correlatos, ou seja, outros mapeamentos/revisões relacionados ao design de interação ou design instrucional em MOOC, identificados durante o MSL, a saber: [Rodrigues et al. 2016a, Sa'don et al. 2014, Zawacki-Richter e Naidu 2016, Rocha e Fantinato 2013].

Rodrigues, Brandão e Brandão (2016) orientaram sua revisão sistemática de modo semelhante à metodologia adotada neste estudo. Contudo, essa revisão foi conduzida de forma iterativa e selecionou bases diferentes das utilizadas neste MSL, foram elas: IEEE Xplore, Google Scholar, Computers \& Education (periódico) e as publicações do Comissão Especial de Informática e Educação da Sociedade Brasileira de Computação (CEIE/SBC). Além disso, esses autores consideraram artigos escritos nas línguas inglesa e portuguesa.

O objetivo da revisão sistemática de Rodrigues, Brandão e Brandão (2016) foi identificar as causas da alta desistência no ensino a distância em estudos publicados entre 2008 e 2015. Nela, os autores buscaram confirmar se a proposta de um modelo para colaboração em pares para apoiar atividades de ensino e aprendizagem na web poderia ser uma estratégia interessante para diminuir as altas taxas de abandono dos cursos online, inclusive cursos MOOC.

Por sua vez, em Sa'don et al. (2014), os autores apresentaram uma revisão sistemática de evidências empíricas relacionadas ao uso de MOOC em instituições de ensino superior (IES). Essa revisão fornece uma visão abrangente sobre as pesquisas realizadas sobre MOOC em IES, concentrando-se entre os anos de 2008 e 2014.

O terceiro estudo, diferentemente dos dois anteriores, é um mapeamento sistemático, cujo objetivo foi destacar as tendências de pesquisa em educação a distância nos 35 anos de publicação da Revista Educação a Distância ${ }^{3}$ [Zawacki-Richter e Naidu 2016]. Esse mapeamento identificou MOOC como o principal tema de pesquisa da educação a distância no ano de 2014; afirmando a importância de se pesquisar e discutir sobre esse tema ainda atual.

No quarto estudo correlato encontrado, os autores utilizaram metodologia semelhante à abordada neste estudo, mas como objetivo distinto. O artigo de Rocha e Fantinato (2013) apresentou resultados de uma revisão sistemática, cujo objetivo foi identificar e avaliar abordagens de Software Product Line (SPL) para Business Process Management (BPM).

\footnotetext{
3 https://mc.manuscriptcentral.com/cdie
} 
Por fim, indicamos como principal contribuição deste estudo em relação aos supracitados a busca e apresentação de trabalhos que abordam contribuições do design instrucional e do design de interação, em especial da articulação entre as áreas, para aplicação em MOOC. Com o intento de obter um design unificado de MOOC que favoreça a aprendizagem e a interação dos estudantes.

\section{Metodologia}

O mapeamento sistemático de literatura, cujos resultados serão apresentados nas seções seguintes, teve por objetivo:

(OE1) identificar abordagens/processos de Design de Interação (DI) para MOOC;

(OE2) identificar abordagens/processos de Design Instrucional (DInst) para MOOC;

(OE3) identificar lacunas de pesquisa nos estudos encontrados.

A metodologia deste mapeamento seguiu as diretrizes propostas por Kitchenham (2004). Em conformidade com essas diretrizes foram seguidas as seguintes etapas: (i) planejamento e definição das questões de pesquisa; (ii) aplicação dos critérios de inclusão e exclusão; (iii) execução do mapeamento e (iv) sumarização dos resultados.

\subsection{Planejamento e questões de pesquisa}

O planejamento consolidou-se com a definição do protocolo de pesquisa para mitigar erros e orientar os pesquisadores durante a execução do mapeamento. No protocolo estão contidas as questões de pesquisa com suas hipóteses relacionadas.

Para atender aos objetivos definidos, foram elaboradas uma questão principal (QP0) e duas questões específicas (QP1 e QP2):

- QP0: Há abordagens, diretrizes ou modelos integrados/articulados de design instrucional e design de interação aplicados a MOOC?

- Rationale: A QP0 foi definida com o propósito de investigar se existem estudos que colaborem com a proposta de abordagem articulada de design indicada por [Garrido 2018].

- Hipótese: Há estudos que proponham abordagens, diretrizes ou modelos integradores de design instrucional e design de interação aplicados a MOOC.

○ Hipótese negativa: Não existem estudos que proponham abordagens, diretrizes ou modelos aplicados a MOOC que integrem design instrucional e design de interação.

- QP1: Quais abordagens, diretrizes ou modelos de design de interação têm sido aplicados a MOOC?

- Rationale: A QP1 foi definida com o propósito de identificar estudos que utilizem abordagens, diretrizes ou modelos de design de interação aplicadas a MOOC.

- Hipótese: Existem estudos que utilizem abordagens, diretrizes, modelos de design de interação aplicados a MOOC.

- QP2: Quais abordagens, diretrizes ou modelos de design instrucional têm sido aplicados a MOOC?

- Rationale: A QP2 foi definida com o propósito de identificar estudos que utilizem abordagens, diretrizes ou modelos de design instrucional aplicadas a MOOC. 
- Hipótese: Durante as buscas nas bases de dados para realização deste MSL serão encontrados estudos que utilizem abordagens, diretrizes ou modelos de design instrucional aplicados a MOOC.

\subsection{Critérios de inclusão e de exclusão}

Os trabalhos que não atenderam a todos os critérios de inclusão ou ao menos um critério de exclusão foram retirados (excluídos) do MSL. Os demais estudos foram mantidos nas etapas subsequentes do mapeamento.

\section{Critérios de Inclusão:}

CI1. Estudo cujo objeto de pesquisa estivesse diretamente relacionado a Massive Open Online Course.

CI2. Estudo que apresentasse prática ou processo de design de interação ou design instrucional.

\section{Critérios de Exclusão:}

CE1. Artigo duplicado.

CE2. Artigo considerado de literatura cinza ${ }^{4}$.

CE3. $\quad$ Artigo com menos de cinco (5) páginas (short paper).

CE4. Estudo secundário (revisão e mapeamento de literatura).

CE5. Artigo indisponível (de acesso restrito ou não encontrados).

CE6. Artigo com insuficiência de conteúdo para os objetivos deste mapeamento.

CE7. Artigo escrito em idioma diferente do inglês.

\subsection{Execução}

Para execução do MSL foram criados dois conjuntos de strings, sendo o primeiro com o uso do acrônimo MOOC e o segundo com o termo Massive Open Online Course, para evitar a inclusão de estudos em que a sigla MOOC não trate sobre o interesse deste MSL. As buscas foram realizadas pela composição do termo MOOC ou Massive Open Online Course em conjunto com os demais termos referentes a design de interação (interaction design) e design instrucional (instructional design), como pode ser visualizado no Quadro 1 e no Quadro 2.

Quadro 1: MOOC e termos relacionados

\begin{tabular}{c} 
MOOC and Interaction Design \\
MOOC and Instructional Design \\
MOOC and Interaction Design and Instructional Design \\
\hline
\end{tabular}

Quadro 2: Massive Open Online Course e termos relacionados

Massive Open Online Course and Interaction Design

Massive Open Online Course and Instructional Design

Massive Open Online Course and Interaction Design and Instructional Design

Foram considerados os estudos publicados até o mês de junho de 2018. Não foi considerado outro ano anterior ao de 2008, pois Grainger (2013) relata que esse foi o ano que o termo MOOC foi criado. Considerando a expressiva quantidade de estudos indexados e relevância

\footnotetext{
${ }^{4}$ Foram considerados de literatura cinza: relatórios (técnicos, preliminar, avançado), teses, position papers (ou ensaios)- documento de posição que apresenta uma opinião discutível sobre uma questão/tema - e white papers conteúdo não revisado que é utilizado para divulgar dados ou ações de sucesso de uma organização.
} 
destas para a área de Ciência da Computação, as buscas ocorreram na seguintes bases: ACM Digital Library $^{5}$, IEEE Xplorer ${ }^{6}$, Scopus ${ }^{7}$ e Science Direct ${ }^{8}$.

Para efeitos de acurácia, optou-se por utilizar as ferramentas de busca avançada oferecidas por cada base, com uso de filtros e adaptações das strings. A adaptação para busca em cada base supracitada pode ser conferida no Apêndice A, situado ao final do artigo.

Após a busca nas bases selecionadas, deu-se seguimento às subetapas de execução do mapeamento ( $1^{\circ}$ filtro). Foram, portanto, aplicados os critérios de exclusão e inclusão. Em seguida, foi realizada a leitura dos títulos, resumo e palavras-chave de cada artigo. Para isso, utilizou-se o Mendeley ${ }^{9}$ como ferramenta de suporte.

Nos artigos em que ocorreu divergência entre os pesquisadores, em nível de doutorado, quanto à adequação aos critérios de inclusão ou de exclusão, foram revisitados em uma subetapa de conciliação para reavaliação cruzada dos estudos divergentes com os professores da pósgraduação em ciência da computação da Universidade Federal da Bahia, aqui chamados de experts.

Nesse momento, cada pesquisador apresentou os motivos de aceitação do artigo para os demais; caso houvesse consenso, o artigo passaria para a próxima fase; caso contrário, seria descartado. Em seguida, esses pareceres foram submetidos aos experts para validação.

Na segunda subetapa de execução do mapeamento, realizou-se a leitura das seções de introdução e conclusão de todos os trabalhos aceitos na fase anterior. Os artigos, cuja leitura da introdução e da conclusão não foram suficientes para o julgamento de aderência ao MSL, foram lidos na íntegra por meio das técnicas de leitura dinâmica, skimming e scanning, por não exigirem leitura precisa e detalhada do texto por completo.

Nos casos em que a divergência de opiniões se manteve, realizou-se nova rodada de reavaliação cruzada. Ressalta-se que todos os pesquisadores envolvidos no MSL são autores deste artigo, sendo dois estudantes em nível de doutorado e dois professores da pós-graduação (com mais de 05 anos de doutoramento).

Com o objetivo de estabelecer um ranking entre os artigos selecionados, considerando sua relevância para o alcance dos objetivos do MSL, foram aplicados os seguintes critérios de qualidade sobre os trabalhos selecionados até esta etapa:

- a estrutura é adequada e de fácil compreensão;

- os objetivos são claramente definidos;

- o problema de pesquisa é claramente definido;

- a metodologia de pesquisa é claramente descrita;

- uma prática de Design de Interação ou Instrucional aplicada a MOOC é apresentada;

- o processo de concepção ou avaliação de um MOOC é descrito;

- o processo de Design de Interação de um MOOC é descrito;

- o processo de Design Instrucional de um MOOC é descrito;

- a avaliação do Design de Interação ou Instrucional do MOOC é apresentada; e

\footnotetext{
${ }^{5}$ https://dl.acm.org/

${ }^{6}$ https://ieeexplore.ieee.org/Xplore/home.jsp

${ }^{7}$ https://www.scopus.com/search/form.uri?display=basic

${ }^{8}$ https://www.sciencedirect.com/

${ }^{9}$ https://www.mendeley.com/
} 
- um guideline ou similar com melhores práticas para concepção de MOOC é apresentado.

Para cada critério de qualidade atribuiu-se uma das seguintes notas: zero (0), meio (0.5) ou um (1). Essas notas foram decorrentes da avaliação dos pesquisadores quanto ao nível de cumprimento do critério de qualidade. Os valores numéricos representam Não (0), Parcial (0.5) e $\operatorname{Sim}(1.0)$.

Estipulou-se nota mínima de seis $(6,0)$ para que o artigo fosse considerado selecionado à fase de extração de dados. Portanto, após aplicar os critérios de qualidade, os estudos que obtiverem nota igual ou superior a seis $(6,0)$, mediante a soma das notas atribuídas por cada critério na classificação, foram selecionados para a fase seguinte, extração de dados.

Na terceira subetapa foi realizada a extração de dados. Nesse momento, os trabalhos foram lidos na íntegra para obtenção dos dados que atendessem aos objetivos do mapeamento sistemático, considerando os critérios de qualidades definidos pelos pesquisadores.

\section{Resultados encontrados}

As buscas nas bases selecionadas retornaram 309 trabalhos (considerando os artigos duplicados). O gráfico da Figura 1 apresenta a quantidade de trabalhos encontrados em cada base, apresentando o percentual em relação ao total. Após retirar os artigos duplicados, restaram 215 trabalhos.

A segunda subetapa de execução do mapeamento (de leitura das seções de introdução e conclusão) resultou inicialmente em 179 artigos. Ao final dessa etapa, após solução das divergências de opinião e reavaliação cruzada, conforme preconiza a metodologia, restaram 43 artigos.

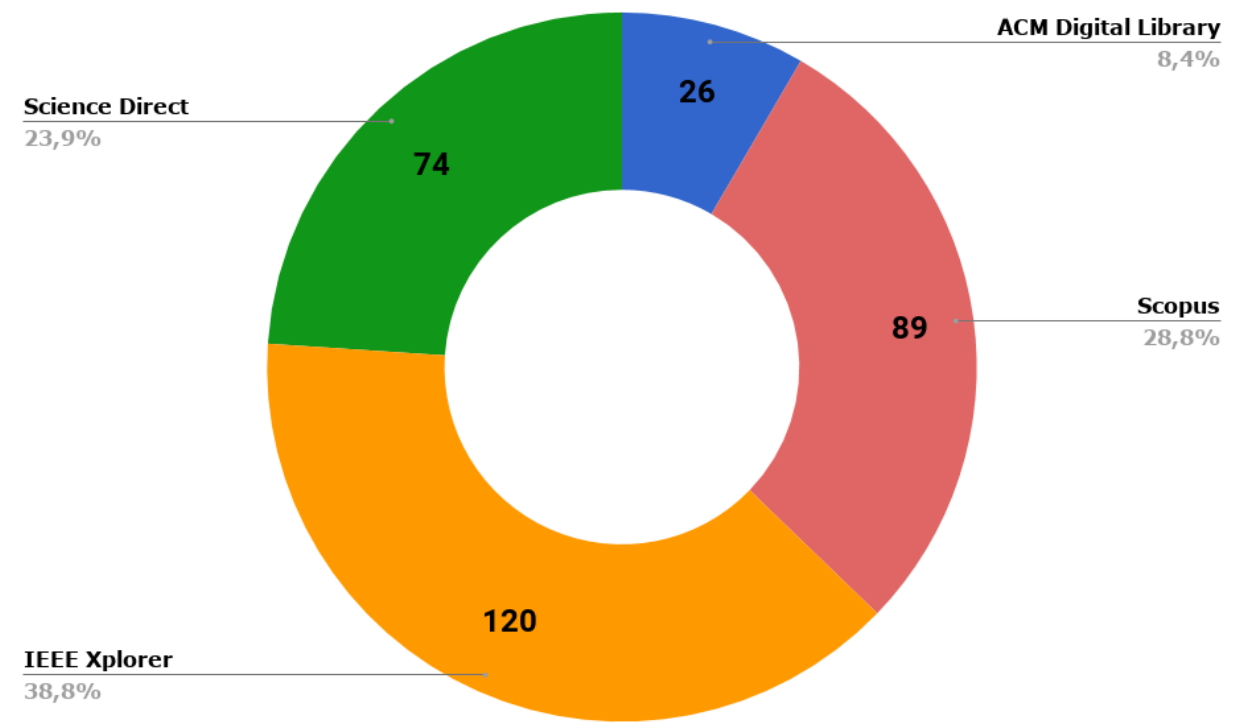

Figura 1: Quantidade de artigos encontrados em cada base - dados brutos.

A Figura 2 destaca o número de artigos encontrados nas buscas iniciais, após aplicação dos critérios de inclusão e de exclusão, e a quantidade final de artigos que passaram à leitura integral e aplicação dos critérios de qualidade. 


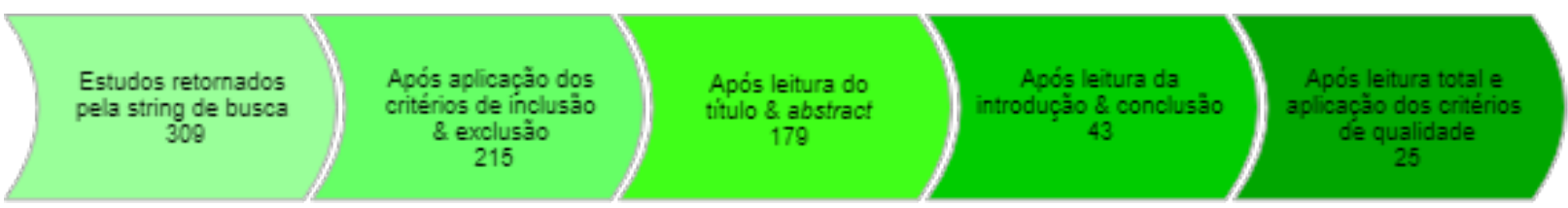

Figura 2: Quantidade de artigos selecionados na realização do MSL.

A Figura 3 apresenta os artigos classificados por ano de publicação. Nota-se que a maior quantidade de publicações ocorreu nos anos de 2016 e 2017, demonstrando que o tema sobre MOOC e Design Instrucional ou Design de Interação é um assunto recorrente. Outro destaque identificado foi a evolução no número de trabalhos desde 2012 até o ano de 2014 e, em seguida, a queda dos estudos encontrados no ano de 2015.

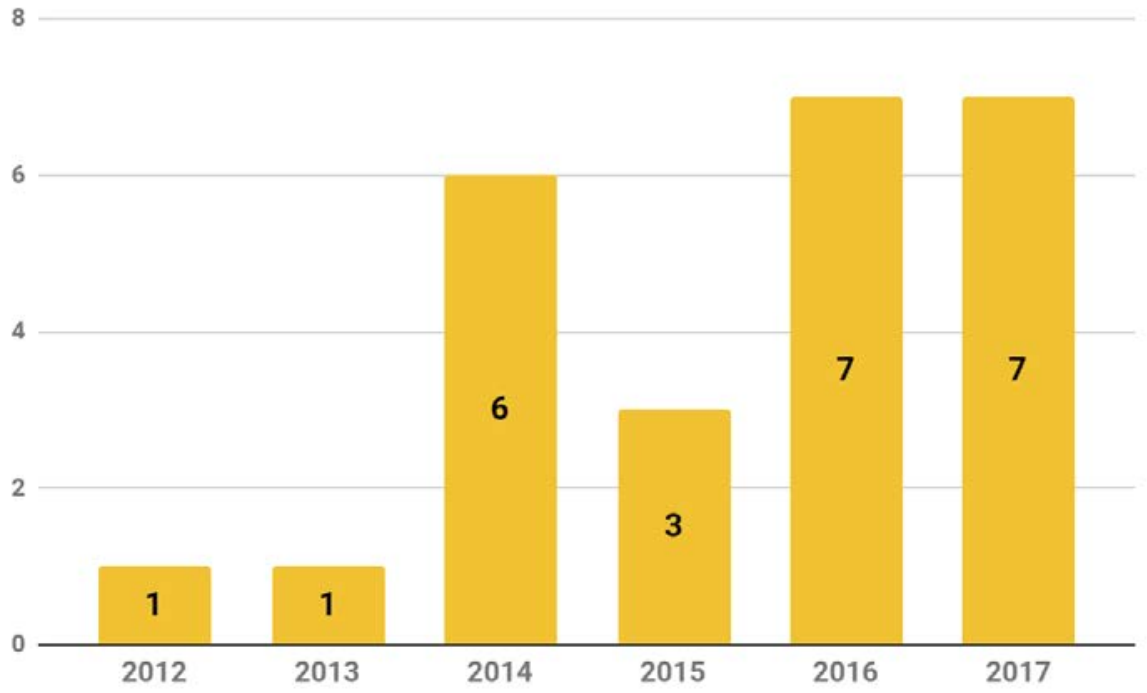

Figura 3: Número de estudos encontrados por ano.

Dos estudos identificados, apenas nove (09) foram publicados em periódicos, os demais (16) foram publicados em conferências. Dentre os trabalhos publicados em conferências, apenas dois foram identificados nos anais da mesma conferência, intitulada Global Engineering Education Conference. Mesmo valor retornado por duas revistas, Computers \& Education e Educational Technology Research and Development, ambas possuindo dois (02) artigos cada indexados em seus anais.

Notou-se que o artigo mais antigo selecionado neste MSL data de 2012. Esse ano ficou mundialmente conhecido, segundo o jornal “The New York Times", como ano dos MOOC [Pappano 2012]. Isso porque neste ano ocorreram diversas iniciativas de suporte metodológico e ferramental a essa nova modalidade de ensino que os MOOC propiciavam, sendo algumas dessas iniciativas conhecidas até hoje, como Coursera ${ }^{10}$ e $\mathrm{EDx}^{11}$.

Com o objetivo de verificar as atualizações de estudos que tratam da temática desse mapeamento, os pesquisadores realizaram em janeiro de 2019 novas buscas nas mesmas bases com as strings de busca apresentadas no protocolo. Apesar de novos achados, nenhum dos estudos

\footnotetext{
10 https://pt.coursera.org/

11 https://www.edx.org/
} 
foi selecionado para a fase de extração por não atenderem aos critérios de inclusão, a maioria foi short papers. Também verificou-se que esses trabalhos não atenderam aos critérios deste MSL.

Os achados (finais), 25 (vinte e cinco) artigos no total, foram agrupados como respostas aos objetivos deste MSL. Dentre os vinte e cinco (25) selecionados para a fase de extração de dados, a maioria trata de design instrucional (84\%). Apenas 16\% trata de design de interação. Acreditamos que o número maior de estudos que tratam de design instrucional em comparação aos de design de interação aplicados a MOOC, possua relação com a natureza educacional dos MOOC; sendo, portanto, alvo de maior interesse do design instrucional (ver Figura 3). Uma vez que o design de interação relaciona-se com qualquer tipo de sistema interativo.

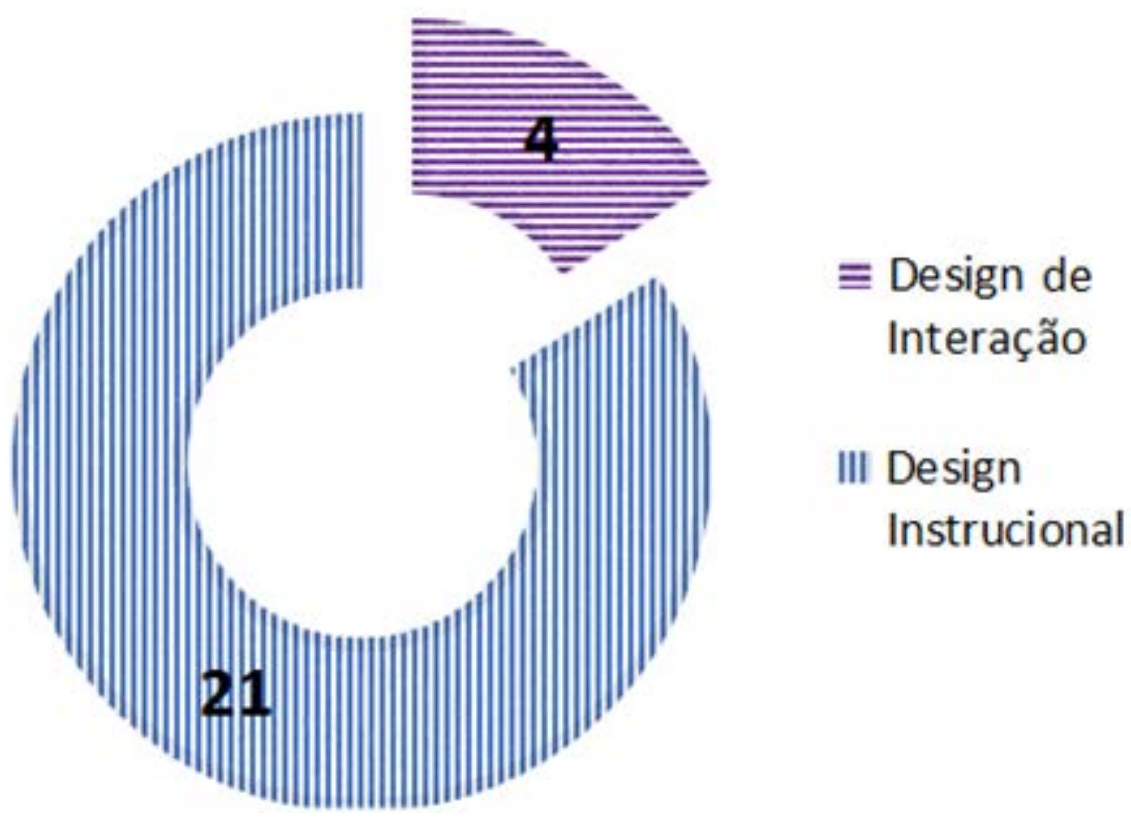

Figura 3: Sumarização estudos fase de extração dos dados.

No Quadro 3 são apresentados os títulos (e autores) dos trabalhos referentes ao Design de Interação. No Quadro 4, daqueles que tratam sobre Design Instrucional aplicado a MOOC. Ressalta-se que esses artigos foram selecionados após a leitura integral e aplicação dos critérios de qualidade. Os quadros encontram-se divididos em três colunas, cada uma composta por um identificador do artigo (ID), título e autores da obra.

Quadro 3: Artigos encontrados sobre Design de Interação

\begin{tabular}{|c|l|l|l|l|}
\hline ID & \multicolumn{1}{|c|}{ Título } & \multicolumn{1}{|c|}{ Autores } & Ano & \multicolumn{1}{|c|}{ Veículo } \\
\hline 01 & $\begin{array}{l}\text { Extending web-based educational } \\
\text { systems with personalised support } \\
\text { through User Centred Designed } \\
\text { recommendation salong the e-learning } \\
\text { life cycle }\end{array}$ & $\begin{array}{l}\text { Olga C. Santosa, Jesus G. } \\
\text { Boticario e Diana Pérez- } \\
\text { Marín }\end{array}$ & 2014 & $\begin{array}{l}\text { Períodico - Science of } \\
\text { Computer Programming }\end{array}$ \\
\hline 02 & $\begin{array}{l}\text { From software engineering to to } \\
\text { courseware engineering }\end{array}$ & $\begin{array}{l}\text { Carlos Delgado Kloos, Ma } \\
\text { Blanca Ibáñez, Carlos } \\
\text { Alario-Hoyos, PedroJ. } \\
\text { Muñoz-Merino, Iria Estévez } \\
\text { Ayres,Carmen Fernández } \\
\text { Panadero e Julio Villena }\end{array}$ & 2016 & $\begin{array}{l}\text { Conferência - Global } \\
\text { Engineering Education } \\
\text { Conference (EDUCON) }\end{array}$ \\
\hline
\end{tabular}




\begin{tabular}{|c|l|l|l|l|}
\hline 03 & $\begin{array}{l}\text { Raising awareness of the accessibility } \\
\text { challenges in mathematics MOOCs }\end{array}$ & $\begin{array}{l}\text { Alexa Ramí rez-Vega, } \\
\text { Francisco Iniesto e } \\
\text { Covadonga Rodrigo }\end{array}$ & 2017 & $\begin{array}{l}\text { Conferência - International } \\
\text { Conference on } \\
\text { Technological Ecosystems } \\
\text { for Enhancing } \\
\text { Multiculturality }\end{array}$ \\
\hline 04 & $\begin{array}{l}\text { Reputation Systems Impact on Help } \\
\text { Seeking in MOOC Discussion Forum }\end{array}$ & $\begin{array}{l}\text { Iris Howley, Gaurav } \\
\text { Tomar, Oliver Ferschke e } \\
\text { Carolyn Penstein Rosé }\end{array}$ & 2017 & $\begin{array}{l}\text { Períodico - IEEE } \\
\text { Transactions on Learning } \\
\text { Technologies }\end{array}$ \\
\hline
\end{tabular}

Quadro 4: Artigos encontrados sobre Design de Instrucional

\begin{tabular}{|c|c|c|c|c|}
\hline ID & Título & Autores & Ano & Veículo \\
\hline 05 & $\begin{array}{l}\text { A proposed framework for an adaptive } \\
\text { learning of Massive Open Online } \\
\text { Courses (MOOCs }\end{array}$ & $\begin{array}{l}\text { Ahmed Alzaghoul e } \\
\text { Edmundo Tovar }\end{array}$ & 2016 & $\begin{array}{l}\text { Conferência - International } \\
\text { Conference on Remote } \\
\text { Engineering and Virtual } \\
\text { Instrumentation (REV) }\end{array}$ \\
\hline 06 & A-Z_ A recipe for a successful MOOC & $\begin{array}{l}\text { Ian Brown, Indie Chung e } \\
\text { Anthony Ho }\end{array}$ & 2016 & $\begin{array}{l}\text { Conferência - International } \\
\text { Conference on e-Learning }\end{array}$ \\
\hline 07 & $\begin{array}{l}\text { Are we all on the same boat_ } \\
\text { coordinating stakeholders for the design } \\
\text { of MOOCs }\end{array}$ & $\begin{array}{l}\text { Carlos Alario-Hoyos, Mar } \\
\text { Pérez-Sanagustín e Carlos } \\
\text { Delgado-Kloos }\end{array}$ & 2014 & $\begin{array}{l}\text { Conferência - European } \\
\text { Conference on Technology } \\
\text { Enhanced Learning }\end{array}$ \\
\hline 08 & $\begin{array}{l}\text { Code yourself and a programar A } \\
\text { bilingual MOOC for teaching computer } \\
\text { science toteenagers }\end{array}$ & $\begin{array}{l}\text { Inés Friss de Kereki e Areti } \\
\text { Manataki }\end{array}$ & 2016 & $\begin{array}{l}\text { Conferência - IEEE } \\
\text { Frontiers in Education } \\
\text { Conference (FIE) }\end{array}$ \\
\hline 09 & $\begin{array}{l}\text { Developing a Computer Programming } \\
\text { MOOC }\end{array}$ & $\begin{array}{l}\text { Natalia Spyropoulou, } \\
\text { Gerasimoula Demopoulou, } \\
\text { Christos Pierrakeas, } \\
\text { Ioannis Koutsonikos e } \\
\text { Achilles Kameas }\end{array}$ & 2015 & $\begin{array}{l}\text { Conferência - International } \\
\text { Conference on } \\
\text { Communication, } \\
\text { Management and } \\
\text { Information Technology }\end{array}$ \\
\hline 10 & $\begin{array}{l}\text { Developing a MOOC experimentation } \\
\text { platform }\end{array}$ & $\begin{array}{l}\text { Vitomir Kovanovi, Srecko } \\
\text { Joksimovi, Philip } \\
\text { Katerinopoulos, } \\
\text { Charalampos } \\
\text { Michail, George Siemens e } \\
\text { Dragan Gasevi }\end{array}$ & 2017 & $\begin{array}{l}\text { Conferência - Seventh } \\
\text { International Learning } \\
\text { Analytics \& Knowledge } \\
\text { Conference }\end{array}$ \\
\hline 11 & $\begin{array}{l}\text { Factors Influencing Learning and } \\
\text { Factors Influencing Persistence }\end{array}$ & $\begin{array}{l}\text { Biyun Huang e Khe Foon } \\
\text { Hew }\end{array}$ & 2017 & $\begin{array}{l}\text { Conferência - International } \\
\text { Conference on Information } \\
\text { System and Data Mining }\end{array}$ \\
\hline 12 & $\begin{array}{l}\text { Five principles for MOOC design_ } \\
\text { With a case study }\end{array}$ & $\begin{array}{l}\text { John R. Drake, Margaret } \\
\text { O'Hara e } \\
\text { Elaine Seeman }\end{array}$ & 2015 & $\begin{array}{l}\text { Conferência - International } \\
\text { Conference on Information } \\
\text { System and Data Mining }\end{array}$ \\
\hline 13 & $\begin{array}{l}\text { Instructional design, facilitation and } \\
\text { perceived learning outcomes - an } \\
\text { exploratory case study of a human } \\
\text { trafficking MOOC }\end{array}$ & $\begin{array}{l}\text { Sunnie Lee Watson, Jamie } \\
\text { Loizzo, William R. Watson, } \\
\text { Chad Mueller, Jieun Lim e } \\
\text { Peggy A. Ertmer }\end{array}$ & 2015 & $\begin{array}{l}\text { Periódico - Journal of } \\
\text { Information Technology } \\
\text { Education: Innovations in } \\
\text { Practice }\end{array}$ \\
\hline 14 & $\begin{array}{l}\text { Instructional Design Models to Support } \\
\text { Collaborative Open Books for Open } \\
\text { Education }\end{array}$ & $\begin{array}{l}\text { Rodés, V., Mustaro, P. N., } \\
\text { Silveira, I. F., Omar, N. e } \\
\text { Ochôa, X. }\end{array}$ & 2016 & $\begin{array}{l}\text { Periódico - Educational } \\
\text { Technology Research and } \\
\text { Development }\end{array}$ \\
\hline 15 & $\begin{array}{l}\text { Instructional quality of Massive Open } \\
\text { Online Courses (MOOCs) }\end{array}$ & $\begin{array}{l}\text { Anoush Margaryan, } \\
\text { Manuela Bianco e Allison } \\
\text { Littlejohn }\end{array}$ & 2014 & $\begin{array}{l}\text { Conferência - International } \\
\text { Conference on Human } \\
\text { Computer Interaction }\end{array}$ \\
\hline 16 & $\begin{array}{l}\text { Learner profiles of attitudinal learning } \\
\text { in a MOOC - An explanatory sequential } \\
\text { mixed methods study }\end{array}$ & $\begin{array}{l}\text { Sunnie Lee Watson, William } \\
\text { R. Watson, Ji Hyun Yu, } \\
\text { Hamdan Alamri, Chad } \\
\text { Mueller }\end{array}$ & 2015 & $\begin{array}{l}\text { Periódico - Computers \& } \\
\text { Education }\end{array}$ \\
\hline 17 & $\begin{array}{l}\text { MOOCocracy_the learning culture of } \\
\text { massive open online courses }\end{array}$ & $\begin{array}{l}\text { Jamie Loizzo e Peggy A. } \\
\text { Ertmer }\end{array}$ & 2017 & $\begin{array}{l}\text { Periódico - Computers \& } \\
\text { Education }\end{array}$ \\
\hline 18 & $\begin{array}{l}\text { MOOCs_Is There an App for That } \\
\text { Expanding Mobilegogy Through an } \\
\text { Analysis of MOOCS and iTunes } \\
\text { University }\end{array}$ & $\begin{array}{l}\text { Patricia A. Machun, } \\
\text { Catherine Trau, Nadia } \\
\text { Zaid, Minjuan Wang e } \\
\text { Jason Ng }\end{array}$ & 2016 & $\begin{array}{l}\text { Periódico - Educational } \\
\text { Technology Research and } \\
\text { Development }\end{array}$ \\
\hline 19 & $\begin{array}{l}\text { Problem-Based Learning in a MOOC - } \\
\text { Exploring an Innovative Instructional } \\
\text { Design at a Large Scale }\end{array}$ & $\begin{array}{l}\text { Daniëlle M.L. Verstegen, } \\
\text { Annemarie Spruijt, Diana }\end{array}$ & 2012 & $\begin{array}{l}\text { Conferência - International } \\
\text { Joint Conferences on Web }\end{array}$ \\
\hline
\end{tabular}




\begin{tabular}{|c|c|c|c|c|}
\hline & & $\begin{array}{l}\text { Dolmans e Jeroen van } \\
\text { Merriënboer }\end{array}$ & & $\begin{array}{l}\text { Intelligence and Intelligent } \\
\text { Agent Technology }\end{array}$ \\
\hline 20 & $\begin{array}{l}\text { Recommendations for the design and } \\
\text { deployment of MOOCs }\end{array}$ & $\begin{array}{l}\text { Carlos Alario-Hoyos, Mar } \\
\text { Pérez-Sanagustín, Carlos } \\
\text { Delgado Kloos e Pedro J. } \\
\text { Muñoz-Merino }\end{array}$ & 2016 & $\begin{array}{l}\text { Conferência - International } \\
\text { Conference on Computer } \\
\text { Supported Education }\end{array}$ \\
\hline 21 & $\begin{array}{l}\text { The Effect of MOOCs Instructional } \\
\text { Design Model-Based on Students' } \\
\text { Learning and Motivation }\end{array}$ & $\begin{array}{l}\text { Eisa rezaei, Esmaeil Zaraii } \\
\text { Zavaraki, Javad Hatami, } \\
\text { Khadijeh Ali Abadi e Ali } \\
\text { Delavar }\end{array}$ & 2014 & $\begin{array}{l}\text { Conferência - International } \\
\text { Conference on } \\
\text { Technological Ecosystems } \\
\text { for Enhancing } \\
\text { Multiculturality. }\end{array}$ \\
\hline 22 & $\begin{array}{l}\text { The mediating effects of germane } \\
\text { cognitive load on the relationship } \\
\text { between instructional design and } \\
\text { students' future behavioral intention }\end{array}$ & $\begin{array}{l}\text { Jamie Costley and } \\
\text { Christopher Lange }\end{array}$ & 2017 & $\begin{array}{l}\text { Periódico - Electronic } \\
\text { Journal of e-Learning }\end{array}$ \\
\hline 23 & The sudden revival of educational video & Jon Baggaley & 2013 & $\begin{array}{l}\text { Conferência - Conference } \\
\text { International Council for } \\
\text { Educational Media (ICEM) }\end{array}$ \\
\hline 24 & $\begin{array}{l}\text { Towards a collaborative pedagogical } \\
\text { model in MOOCs }\end{array}$ & $\begin{array}{l}\text { Iván Claros, Antonio } \\
\text { Garmendía, Leovy } \\
\text { Echeverría e Ruth Cobos }\end{array}$ & 2014 & $\begin{array}{l}\text { Conferência - IEEE Global } \\
\text { Engineering Education } \\
\text { Conference (EDUCON) }\end{array}$ \\
\hline 25 & $\begin{array}{l}\text { What Drives a Successful MOOC_An } \\
\text { Empirical Examination of Criteria to } \\
\text { Assure Design Quality of MOOCs }\end{array}$ & $\begin{array}{l}\text { Ahmed Mohamed Fahmy } \\
\text { Yousef, Mohamed Amine } \\
\text { Chatti, Ulrik Schroeder e } \\
\text { Marold Wosnitza }\end{array}$ & 2014 & $\begin{array}{l}\text { Conferência - IEEE 14th } \\
\text { International Conference } \\
\text { on Advanced Learning } \\
\text { Technologies }\end{array}$ \\
\hline
\end{tabular}

Na próxima subseção as questões deste MSL serão respondidas por meio das inferências construídas durante a leitura integral dos artigos.

\subsection{Há abordagens, diretrizes ou modelos integrados de Design Instrucional e Design de Interação aplicados a MOOC?}

O MSL confirmou a hipótese nula de que não há (não foram encontrados) estudos que concebam ou executem abordagens, diretrizes ou modelos integradores de design instrucional e design de interação aplicados a MOOC. Ao menos, esses trabalhos não foram encontrados.

As buscas nas bases de dados selecionadas não retornaram trabalhos que relacionassem (integrassem) design instrucional e design de interação aplicado-os em MOOC; logo, não foram encontradas abordagens, diretrizes ou modelos com tal característica integrativa. Logo, identificou-se uma lacuna de pesquisa.

Ressalta-se que o resultado do design instrucional é compreendido como parte integrante da interação do estudante com/no curso [Filatro 2008]. Entretanto, a ausência de estudos que relacionem esses campos de design, indica um desafio para a concepção de processos (abordagens) híbridos(as) de design para MOOC.

\subsection{Quais abordagens, diretrizes ou modelos de Design de Interação têm sido aplicados a MOOC?}

O MSL confirmou a hipótese de que há estudos que utilizam abordagens, diretrizes ou modelos de Design de Interação aplicados a MOOC. Foram identificados quatro (4) trabalhos que versam sobre essa questão.

Um dos estudos indica a dificuldade de tornar os MOOC tecnologicamente acessíveis a estudantes com deficiência, especialmente deficiência visual [Ramírez-Vega et al. 2017]. Os autores realizaram ensaios de acessibilidade nas páginas de cada curso, utilizando técnicas manuais e automáticas de avaliação. Em suas conclusões, os autores apresentam diretrizes gerais de acessibilidade para cursos e plataformas de MOOC, especialmente para as últimas que 
apresentam os maiores problemas nesse aspecto. Eles também evidenciam a necessidade de revisão dos processos de design instrucional seguidos pelas universidades provedoras de conteúdo. A ausência de informações mais precisas sobre como os conteudistas querem apresentar as instruções no conteúdo, dificulta a criação de legendas ou representação das descrições e transcrições em áudio. Isso, portanto, cria barreiras para o acesso universal ao curso oferecido.

Outro trabalho destacado é o estudo que propôs uma abordagem de design centrado no usuário utilizando um sistema de recomendação [Santos et al. 2014]. Essa adaptação baseou-se nas respostas de usuários durante seu levantamento de perfil em um ambiente de e-learning. Para avaliar o Design Instrucional realizado, os autores utilizaram o feedback dos usuários como validador.

A preocupação com o ciclo de vida dos materiais didáticos criados e disponibilizados nos MOOC, com foco na manutenção, qualidade e diversidade do público alvo, é o principal aspecto de relevância para um dos estudos extraídos [Kloos et al. 2016]. Os autores expressaram o objetivo de conceber um conteúdo instrucional que fosse eficaz (apropriado); eficiente (ensinando o sujeito a usar recursos tanto quanto possível); e utilizável (sendo fácil de usar e aprender). Para o escopo de criar um material de ensino com todo esse potencial, eles sugerem a adoção de fundamentos e ferramentas de engenharia de software (ferramentas CASE ${ }^{12}$ ).

Dos artigos selecionados na etapa de extração, quatro (4) tratam prioritariamente sobre design de interação aplicado a MOOC, enfatizando apenas critérios relacionados a usabilidade e acessibilidade. Entretanto, nenhum deles apresentou resultados sobre a qualidade da metacomunicação (comunicabilidade sistema-usuário), focalizando apenas em aspectos como usabilidade e acessibilidade.

\subsection{Quais abordagens, diretrizes ou modelos de Design de Instrucional têm sido aplicados a MOOC?}

Vinte e um (21) trabalhos contribuíram na resposta à questão. Quantidade maior em comparação aos estudos que trataram de design de interação aplicado a MOOC. Esses trabalhos apresentaram, portanto, propostas, abordagens ou modelos de design instrucional aplicados a MOOC. Isso confirmou a hipótese de haver estudos que utilizem abordagens, diretrizes ou modelos de design instrucional aplicados a MOOC.

Uma evidência relevante apontada por um dos estudos é que a utilização de modelos para criação do design instrucional de um MOOC influencia a qualidade de aprendizado, como também influencia a taxa de abandono de seus estudantes [Delavar 2017].

Delavar (2017) investigou as características únicas dos MOOC, focalizando nas evidências do design instrucional sob o aspecto qualitativo e apresentou um modelo dividido em nove (09) categorias principais e 22 subcategorias com resultados de eficácia da aprendizagem e medição da motivação dos estudantes. Por fim, concluiu que para um design eficaz é necessário diversificar as atividades dos estudantes, incentivar a participação dos estudantes e preocupar-se com o contexto dos estudantes para que possam transferir conhecimento de sua experiência real em um MOOC.

\footnotetext{
12 Ferramentas CASE (Computer Aided System Engineering) é um conjunto de software com o objetivo de automatizar processos de engenharia de software, provendo suporte básico na sua concepção, construção, manutenção e documentação de sistemas [Loiola e Rocha 2002].
} 
Outro estudo desenvolvido por Alario-Hoyos, Pérez-Sanagustín e Delgado-Kloos (2014) apresenta o MOOC Canvas, artefato utilizado como ferramenta epistêmica para os stakeholders ${ }^{13}$ efetuarem o design de um MOOC. Esse canvas foi construído por 27 (vinte e sete) participantes em três workshops, dividido em 11 (onze) quadrantes com objetivos/recursos do MOOC a ser desenvolvido. O uso desse artefato pode favorecer o diálogo da equipe responsável pelo design instrucional do MOOC [Garrido 2018].

Outro estudo enfatizou que o Design Instrucional deve incorporar uma variedade de estratégias para chamar a atenção e interesse dos estudantes, de modo a estimular a curiosidade na investigação [Huang e Hew 2017]. Para isso, os autores utilizaram o modelo de design instrucional Attention, Relevance, Confidence and Satisfaction ${ }^{14}$ (ARCS) para alcançar o objetivo de engajar os estudantes em um curso MOOC.

\subsection{Sumarização dos resultados}

Os artigos identificados neste MSL, ainda que tratem de áreas distintas, DI e DInst, convergem em diversos aspectos. Algumas ferramentas propostas têm papel fundamental na interação estudante-sistema, como o instrumento para ajuda por pares, Quick Helper MOOC [Howley et al. 2017]. Entretanto, os autores relatam seu impacto somente no aspecto instrucional, sem relacionar os seus reflexos na qualidade da interação dos e entre os estudantes.

Outros trabalhos encontrados propõem integrar ferramentas computacionais em MOOC, como [Santos et al. 2014, Rodes et al. 2014]. Entretanto, a análise sobre o impacto dessa integração é parcial, ou sob a ótica da interação ou sob a ótica da instrução.

Qualquer artefato computacional inserido no ambiente MOOC poderá impactar no percurso educacional e na interação dos estudantes-usuários, como percebido nesse MSL. Sendo necessário analisar sua inserção de forma integrada desde o planejamento do artefato. Entretanto, os trabalhos investigados neste MSL são parciais em suas propostas ou práticas, indicando atuação restrita a um das áreas, design de interação ou design instrucional.

\subsection{Ameaças à validade}

Este estudo considerou artigos publicados em importantes bancos de dados de Ciência da Computação. Obviamente, vários repositórios de artigos científicos estavam fora do nosso escopo. Embora as bases utilizadas sejam confiáveis, a seleção das bases de dados utilizadas é uma ameaça à validade em relação à generalização.

O resultado do MSL pode possuir viés dos pesquisadores. Para mitigar essa ameaça, os pesquisadores seguiram um protocolo que indica como o processo de metodologia deve ser realizado.

Outras potenciais ameaças que foram identificadas são: (i) cobertura da literatura existente. Mesmo que não fosse garantida a cobertura completa do material publicado, a amostra dos estudos retornados pela string de busca é suficientemente representativa para produzir resultados confiáveis sobre a articulação do design de interação com o design instrucional aplicado ao MOOC; (ii) relação do estudo ao impacto dos critérios de qualidade. O uso de uma pontuação para indicar a qualidade dos artigos pode ser um ponto questionador. Nesse sentido, adotou-se critérios de qualidade claros, de acordo com os itens mínimos necessários para chegarmos a uma resposta para as questões de pesquisa.

\footnotetext{
13 Partes interessadas de um projeto.

14 Atenção, Relevância, Confiança e Satisfação (Grifo nosso)
} 


\section{Considerações e trabalhos futuros}

Neste artigo foram apresentados os resultados de um MSL, cujo objetivo foi identificar estudos e pesquisas primárias referentes a práticas de Design de Interação e de Design Instrucional aplicados à MOOC. Ademais, projetamos a conexão entre abordagens, métodos, técnicas, ferramentas de ambos para que possam favorecer a aprendizagem e a interação dos estudantes em MOOC.

Diante dos resultados apresentados neste artigo, constatou-se uma lacuna de pesquisa a ser explorada: o design integrado (interação e instrucional) para MOOC. Portanto, são necessários estudos que permitam a articulação teórica e metodológica entre design de interação e design instrucional em MOOC.

Um dos achados do MSL, o MOOC Canvas em Alario-Hoyos, Pérez-Sanagustín e Delgado-Kloos (2014), serviu de suporte para a concepção de um curso MOOC entre stakeholders co-localizados. Para que isso fosse possível, ocorreram adaptações no MOOC Canvas e o uso de ferramentas computacionais em nuvem, como relatado em Garrido, Do Rêgo e Matos (2018).

Outro aspecto relevante, ignorado nos estudos identificados, é a discussão da importância da comunicabilidade em ambientes educacionais como os MOOC. Investigações de Do Rêgo, Garrido e Matos (2017) apontam para importância de analisar aspectos da metacomunicação (metamensagem) em interfaces de ambientes MOOC, por causa da pluralidade de público e importância de uma comunicação eficiente entre designers, inclusive educacionais, e estudantes (usuários) durante a interação.

Em Do Rêgo, Garrido e Matos (2018), os autores reafirmam a importância de tratar rupturas de comunicabilidade (breakdowns) ao adaptar o Moodle ${ }^{15}$ para uso como MOOC, cujo principal objetivo foi favorecer-se da familiaridade dos estudantes com esse(a) ambiente/interface. O design de interação, sob o aspecto da comunicabilidade, é elemento fundamental à construção de um MOOC, uma vez que o uso e o percurso instrucional dos estudantes também serão impactados pela qualidade desse design.

Para trabalhos futuros, espera-se que as ferramentas, modelos, métodos, técnicas ou práticas de/para design de MOOC encontradas nesse MSL possam ser replicadas de forma individualizada ou conjunta para auxiliar na construção de uma proposta híbrida de design que articule o design de interação com o design instrucional para MOOC.

\section{Agradecimentos}

Agradecemos aos membros do Grupo de Pesquisa e Extensão em Informática, Educação e Sociedade - Onda Digital e a Fundação de Amparo à Pesquisa do Estado da Bahia (FAPESB) pelo financiamento desta pesquisa.

\section{Referências}

Alario-Hoyos, C., Pérez-Sanagustín, M., Kloos, C. D., e Muñoz-Merino, P. J. (2014). Recommendations for the design and deployment of moocs: insights about the mooc digital education of the future deployed in miríadax. Proceedings of the Second International

${ }^{15}$ AVA (Ambiente Virtual de Aprendizagem) amplamente utilizado no Brasil em diversas instituições de ensino 
Conference on Technological Ecosystems for Enhancing Multiculturality, pp. 403-408. DOI: http://dx.doi.org/10.1145/2669711.2669931 [GS Search]

Baloi, J. A. (2009). A concepção da educação democrática na obra "Democracia e Educação" de Jonh Dewey. Monografia - Faculdade de Filosofia e Ciências Humanas da Universidade São Tomás de Moçambique, Maputo, [s. n.]. [GS Search]

Baturay, M. H. (2015). An overview of the world of moocs. Procedia-Social and Behavioral Sciences, v. 174, pp. 427-433. DOI: https://doi.org/10.1016/j.sbspro.2015.01.685 [GS Search]

Boall, H., Chaves, M., e Stallivieri, L. (2015). Os moocs e o processo de internacionalização das instituições de ensino superior. XV Colóquio Internacional de Gestão Universitária -CIGU INPEAU/Universidade Federal de Santa Catarina, [s. n.]. [GS Search]

Dal Forno, J. P., e Knoll, G. F. (2013). Os moocs no mundo: um levantamento de cursos online abertos massivos. Nuances: estudos sobre Educação, v. 24, n .3, pp. 178-194. [GS Search]

Delavar, A. (2017). The effect of moocs instructional design model-based on student's learning and motivation. Man In India, v. 97, n. 11, pp. 115-126. [GS Search]

Do Rêgo, B. B., Garrido, F., e Matos, E. (2017). Identifying influences of the quality of interaction on dropout rates of mooc: preliminary results. In Simpósio Brasileiro de Informática na Educação-SBIE. Recife -PE. Anais Congresso Brasileiro de Informática na Educação - CBIE, Sociedade Brasileira de Computação, Porto Alegre, v. 28, n. 1, pp. 17661768. DOI: http://dx.doi.org/10.5753/cbie.sbie.2017.1766 [GS Search]

Do Rêgo, B. B., Garrido, F. A., e de Souza Matos, E. (2018). Moodle como ambiente mooc:orientações para o redesign de interação. RENOTE - Revista Novas Tecnologias na Educação , v. 16, n. 1, pp. 1-10. DOI: https://doi.org/10.22456/1679-1916.86050 [GS Search]

Fassbinder, A., Delamaro, M. E., e Barbosa, E. F. (2014). Construção e uso de moocs: uma revisão sistemática. In Simpósio Brasileiro de Informática na Educação-SBIE. Recife -PE. Anais Congresso Brasileiro de Informática na Educação - CBIE, Sociedade Brasileira de Computação, Porto Alegre, v. 25, n. 1, pp. 332-341. DOI: http://dx.doi.org/10.5753/cbie.sbie.2014.332 [GS Search]

Filatro, A. (2008). Design instrucional na prática. Pearson Education do Brasil. São Paulo. [GS Search]

Filatro, A., \& Piconez, S. C. B. (2004). Design instrucional contextualizado. São Paulo: Senac. [GS Search]

Garrido, F., do Rêgo, B. B., e Matos, E. (2018). Design instrucional orientado a artefatos: uma abordagem participativa e distribuída. In Simpósio Brasileiro de Informática na EducaçãoSBIE. Fortaleza -CE. Anais Congresso Brasileiro de Informática na Educação - CBIE, Sociedade Brasileira de Computação, Porto Alegre, v. 29, n. 129, pp. 258-268. DOI: http://dx.doi.org/10.5753/cbie.sbie.2018.258 [GS Search]

Garrido, F. A. (2018). Uma Abordagem Unificada de Design para Modelagem da Comunicação e Instrução para MOOC. Dissertação de Mestrado em Ciência da Computação. Universidade Federal da Bahia - UFBA.

Grainger, B. (2013). Introduction to MOOCs: avalanche, illusion or augmentation. Policy Brief - UNESCO (july). Institute for Information Technologies in Education. (URL:http://iite.unesco.org/pics/publications/en/files/3214722.pdf). [GS Search] 
Howley, I., Tomar, G. S., Ferschke, O., e Rose, C. P. (2017.). Reputation systems impact on help seeking in mooc discussion forums. IEEE Transactions on Learning Technologies, n. 1, pp. 1-14. DOI: http://dx.doi.org/10.1109/TLT.2017.2776273 [GS Search]

Huang, B., e Hew, K. F. (2017). Factors influencing learning and factors influencing persistence: A mixed-method study of mooc learners' motivation. Proceedings of the 2017 International Conference on Information System and Data Mining, , Charleston, SC, USA. ACM. pp. 103110. DOI:http://dx.doi.org/10.1145/3077584.3077610 [GS Search]

Kitchenham, B. (2004). Procedures for performing systematic reviews. Keele, UK, Keele University, v. 33, pp. 1-26. [GS Search]

Kloos, C. D., Ibáñez, M. B., Alario-Hoyos, C., Muñoz-Merino, P. J., Ayres, I. E., Panadero, C. F., e Villena, J. (2016). From software engineering to courseware engineering. In 2016 IEEE Global Engineering Education Conference (EDUCON), pp. 1122-1128. DOI: http://dx.doi.org/10.1109/EDUCON.2016.7474695 [GS Search]

Loiola, E., e Rocha, M. C. F. (2002). Aprendizagem no Processo de Seleção de Ferramenta CASE para o Estado da Bahia. Revista de Administração Contemporânea, v. 6, n. 2, pp. 145-166. DOI: http://dx.doi.org/10.1590/S1415-65552002000200009 [GS Search]

Löwgren, J. (2013). Interaction design-brief intro. The Encyclopedia of Human Computer Interaction, 2nd Ed.”. Mads Soegaard and Rikke Friis Dam (Eds.). The Interaction Design Foundation, Aarhus, Dinamarca, Chapter $1 . \quad$ (URL: http://www.interactiondesign.org/encyclopedia/interaction_design.html). [GS Search]

Matta, C. E., e Figueiredo, A. P. S. (2013). Mooc: transformação das práticas de aprendizagem. $X$ Congresso Brasileiro de Ensino Superior a Distância, v. 10, pp. 1-15. DOI: http://dx.doi.org/10.13140/RG.2.1.1392.8086 [GS Search]

Mattar, J. (2013). Fóruns de discussão em educação à distância: Moodle, facebook e redu. Tecnologia Educacional. Revista Da Associação Brasileira De Tecnologia Educacional, v. 202, pp. 6-17. [GS Search]

Pappano, L (2012). The year of the mooc. The New York Times, v. 2, n. 12, pp. 1-7. [GS Search]

Ramírez-Vega, A., Iniesto, F., e Rodrigo, C. (2017). Raising awareness of the accessibility challenges in mathematics moocs. Proceedings of the 5th International Conference on Technological Ecosystems for Enhancing Multiculturality, n. 92, pp. 1-8. DOI: https://doi.org/10.1145/3144826.3145435 [GS Search]

Rocha, R. d. S., e Fantinato, M. (2013). The use of software product lines for business process management: A systematic literature review. Information and Software Technology, v. 55, n. 8, pp. 1355-1373. DOI: https://doi.org/10.1016/j.infsof.2013.02.007 [GS Search]

Rodés, V., Mustaro, P. N., Silveira, I. F., Omar, N., e Ochôa, X. (2014). Instructional design models to support collaborative open books for open education. In Proceedings of the XV International Conference on Human Computer Interaction, [s. n], ACM. DOI: doi.org/10.1145/2662253.2662346 [GS Search]

Rodrigues, P. A., Brandão, L. d. O., e Brandão, A. A. F. (2016). Let us learn together!: Do complementary abilities foster pair collaboration in web-based learning? Frontiers in Education Conference (FIE), IEEE, pp. 1-8. DOI: http://dx.doi.org/10.1109/FIE.2016.7757375 [GS Search] 
Rodrigues, R. L., Ramos, J. L. C., Silva, J. C. S., e Gomes, A. S. (2016). Discovery engagement patterns moocs through cluster analysis. IEEE Latin America Transactions, v. 14, n. 9, pp. 4129-4135. DOI: http://dx.doi.org/10.1109/TLA.2016.7785943 [GS Search]

Rogers, Y., Sharp, H., e Preece, J. (2013). Design de interação: além da interação humanocomputador. Bookman. [GS Search]

Sa'don, N. F., Alias, R. A., \& Ohshima, N. (2014). Nascent research trends in moocs in higher educational institutions: A systematic literature review. Web and Open Access to Learning (ICWOAL), 2014 International Conference on, pp. 1-4. DOI: http://dx.doi.org/10.1109/ICWOAL.2014.7009215 [GS Search]

Santos, O. C., Boticario, J. G., e Pérez-Marín, D. (2014). Extending web-based educational systems with personalised support through user centred designed recommendations along the e-learning life cycle. Science of Computer Programming, v. 88, pp .92-109. DOI: http://dx.doi.org/10.1016/j.scico.2013.12.004 [GS Search]

Zawacki-Richter, O., e Naidu, S. (2016). Mapping research trends from 35 years of publications in distance education. Distance Education, v. 37, n. 3, pp. 245-269. DOI: https://doi.org/10.1080/01587919.2016.1185079 [GS Search] 


\section{Apêndice A - Strings de busca por base}

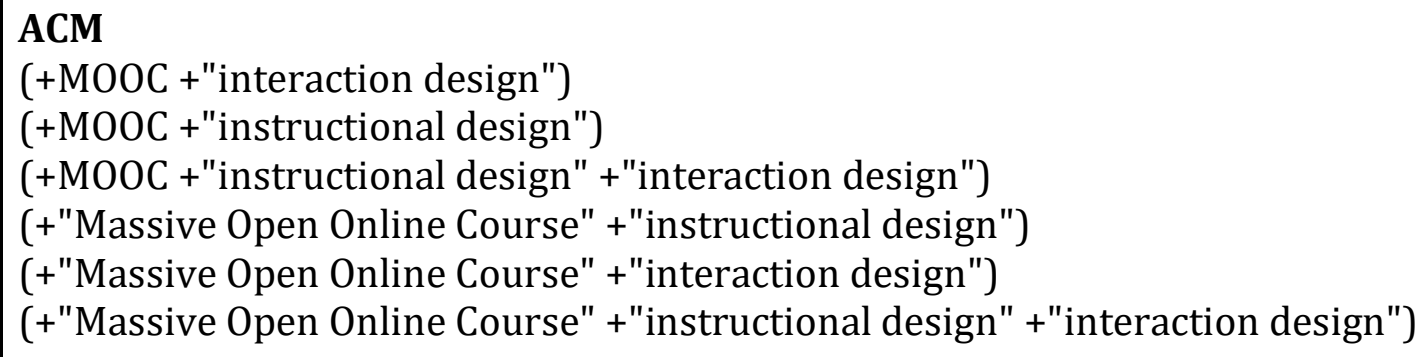

\section{IEEE}

(((MOOC) AND interaction design") AND "instructional design")

((MOOC) AND "instructional design")

((MOOC) AND "interaction design")

((("massive open online course") AND "interaction design") AND "instructional design")

(("massive open online course") AND "interaction design")

(("massive open online course") AND "instructional design")

\section{Science Direct}

(MOOC) and ("interaction design")

(MOOC) and ("instructional design")

(MOOC) and ("interaction design") and ("instructional design")

("massive open online course") and ("instructional design")

("massive open online course") and ("interaction design")

("massive open online course") and ("interaction design") and ("instructional design)

\section{SCOPUS}

TITLE-ABS-KEY ("massive open online course" AND "instructional design")

TITLE-ABS-KEY ("massive open online course" AND "interaction design")

TITLE-ABS-KEY ("massive open online course" AND "interaction design" AND

"instructional design")

TITLE-ABS-KEY (mooc AND "interaction design" AND "instructional design")

TITLE-ABS-KEY (mooc AND "instructional design")

TITLE-ABS-KEY (mooc AND "interaction design") 